\title{
EL IMPACTO DEL CULTIVO, EL ABANDONO \\ Y LA INTENSIFICACIÓN DE LA AGRICULTURA \\ EN LA PÉRDIDA DE AGUA Y SUELO. EL EJEMPLO DE \\ LA VERTIENTE NORTE DE LA SERRA GROSSA EN EL ESTE PENINSULAR
}

\author{
A. CERDÀ ${ }^{(1)^{*}}$, A. GIMÉNEZ MORERA ${ }^{(2)}$, M. BURGUET $^{(3)}$, \\ V. ARCENEGUI ${ }^{(4)}$, F. A. GONZÁLEZ PEÑALOZA ${ }^{(1)}$, \\ F. GARCÍA-ORENES ${ }^{(4)}$, P. PEREIRA ${ }^{(5)}$ \\ ${ }^{1}$ SEDER-Soil Erosion and Degradation Research Group. \\ Departament de Geografia. Universitat de València, \\ Blasco Ibàñez, 28, 46010-Valencia. \\ ${ }^{2}$ Departamento de Economía y Ciencias Sociales, \\ Escuela Politécnica Superior de Alcoy, \\ Universidad Politécnica de Valencia, \\ Paseo del Viaducto, 1, 03801-Alcoy, Alicante. \\ ${ }^{3}$ Instituto de Agricultura Sostenible (IAS-CSIC). \\ Av. Menéndez Pidal s/n. Campus Alameda del Obispo. \\ Apartado 4084, 14080-Córdoba. \\ ${ }^{4}$ GEA (Grupo de Edafología Ambiental). \\ Departamento de Agroquímica y Medio Ambiente, \\ Universidad Miguel Hernández. \\ Avda. de la Universidad, s/n, 03202-Elche, Alicante. \\ ${ }^{5}$ Department of Environmental Policy Mykolas Romeris University, \\ Ateities g. 20, LT-08303 Vilnius, Lituania.
}

\begin{abstract}
RESUMEN. El abandono del campo a lo largo del siglo XX supuso una intensa transformación paisajística en las zonas de montaña en toda la Península Ibérica. En algunos casos, y tras 50 años de abandono, la actividad agrícola ha regresado con explotaciones comerciales y con una intensificación de la actividad agrícola. En el Este Peninsular, tras el abandono de olivares, viñedos y cereales, se ha producido en las dos últimas décadas la expansión de la citricultura intensiva en ladera. Mediante transectos geomorfológicos y experimentos con lluvias simuladas se ha cuantificado el impacto del cultivo tradicional de secano de los años 50, el abandono y posterior recuperación, y la intensificación de la explotación agrícola sobre los procesos y formas de erosión en la vertiente norte de la Serra Grossa, al sur de la provincia de Valencia. Se ha comprobado que los campos de cítricos presentan el mayor número de regueros y cárcavas. Estas morfologías erosivas no existían en el cultivo tradicional por el
\end{abstract}


laboreo, y cuando se formaron en los campos abandonados pronto fueron controlados por el crecimiento de la vegetación. El abandono de los cultivos redujo la pérdida de suelo, pero la citricultura intensiva ha vuelto a reactivar los procesos erosivos hasta tasas que superan en varios órdenes de magnitud el abandono (x4) y que multiplican por 17 las cuantificadas en la agricultura tradicional de secano. El laboreo del secano tradicional reducía las escorrentías, pero aumentaba la concentración de sedimentos de la arroyada.

The impact of the farming, abandonment and agricultural intensification on loss of water and soil. The example of the northern slopes of the Serra Grossa, Eastern Spain

\begin{abstract}
Land abandonment throughout the twentieth century led to an intense landscape transformation in the mountain areas of the Iberian Peninsula. In some cases, and after 50 years of abandonment, agriculture returned with the development of commercial farms and the intensification of the agrarian activities. In the Easter Iberian Peninsula, following the abandonment of olive groves, vineyards and cereals during the 50's, has been in the past two decades the expansion of intensive citrus production on sloping terrain. Geomorphological transects and simulated rainfall experiments have quantified the impact of traditional rainfed cultivation of the 50's, abandonment, and the intensification of farming on the processes and landforms of erosion on the northern slopes of the Serra Grossa, south of the province of Valencia. It was found that the citrus groves have the highest number of rills and gullies. These erosive morphologies were not in the traditional crop tillage, and when they formed in the abandoned fields were soon controlled by the growth of vegetation. The abandonment of the crops reduced the soil losses, but the intensification of the agriculture with the citrus production has accelerated the soil erosion rates that exceed by several orders of magnitude $(x 4)$ the soil erosion rates measured during the abandonment and multiply by 17 the quantified traditional rainfed agriculture soil losses. Tillage of traditional rainfed orchards reduced runoff, but increased the concentration of sediments of the surface wash.
\end{abstract}

Palabras clave: abandono, cultivo, intensificación, cítricos, erosión.

Key words: land abandonment, crop, intensification, citrus, erosion.

Enviado el 30 de septiembre de 2011

Aceptado el 18 de noviembre de 2011

*Correspondencia: SEDER-Soil Erosion and Degradation Research Group. Departament de Geografia. Universitat de València, Blasco Ibàñez, 28, 46010-Valencia. E-mail: artemio.cerda@uv.es 


\section{Introducción}

Las zonas de media montaña de la costa mediterránea de la Península Ibérica fueron abandonadas por sus habitantes a lo largo de los primeros 60 años del siglo XX como consecuencia del rápido e intenso cambio económico que llevó a la población a zonas litorales y urbanas, o al norte de Europa (Garrabou et al., 1986; Cruz, 1990; McNeill, 1992; Collantes, 2004). Esa masiva migración dejo las zonas de montaña sin mano de obra para mantener la milenaria actividad agropecuaria (Lasanta, 1989; Douglas, 1994; Collantes, 2001). Así, los suelos agrícolas volvieron a ver crecer las herbáceas, a las que seguirían los matorrales, y los primeros pies de árboles (Debussche y Lepart, 1992; Cerdà, 1996; Ne'eman y Izhaki,1996; García Fayos y Verdú, 1998; Bonet, 2004) lo que dio lugar a más biomasa (Poyatos et al., 2003). Esto contribuyó a cambios substanciales en las propiedades de los suelos (Cerdà, 1997, 1998; Cammeraat y Imeson, 1999; Zavala et al., 2010). Y con la vegetación cambiaron los procesos hidrológicos y geomorfológicos (Ruiz-Flaño et al., 1991; Cerdà, 1997; García-Ruiz y Valero, 1998). Algo parecido ocurrió con las zonas de pastos y de aprovechamientos de la biomasa, donde el abandono permitió una recuperación vegetal extraordinaria (Debussche et al., 1999; Vicente et al., 2001; Koulouri y Giourga, 2007).

Distintos estudios han permitido comprobar cómo el abandono da como resultado una revegetación natural, si bien en ocasiones alterada por repoblaciones, que ha desencadenado una reducción de los sedimentos y aguas disponibles en los cauces (GarcíaRuiz et al., 2005; Lasanta et al., 2006). Esa vegetación natural sólo es alterada por los incendios forestales, que producen un incremento de la pérdida de suelo y aguas durante los años post-incendio pero que finalmente permiten la continuidad de la recuperación vegetal y edáfica al ser controladas las pérdidas de suelo por la vegetación (Cerdà, 1998; Cerdà y Lasanta, 2005). Ese control de la exportación de materiales afecta también a las pérdidas de solutos (Lasanta y Cerdà, 2005).

En las últimas dos décadas, y partiendo desde el litoral, se ha producido una colonización de las zonas abandonadas por los nuevos cultivos intensivos. No es un proceso generalizado, pero si relevante en la agricultura española, ya que estos cultivos representan la agricultura moderna, tecnificada y más rentable, y su capacidad de transformación del paisaje hace que sus alteraciones sean rápidas e intensas. Esta intensificación de la agricultura, debido a su elevada productividad, hace que el territorio afectado sea menor que el que ocupaba la agricultura tradicional de secano. No obstante su impacto allí donde se produce es mucho mayor por la maquinaria pesada utilizada, los fertilizantes químicos de síntesis, los herbicidas y pesticidas, la aparición de estrategias de producción puramente industriales, y los materiales sintéticos (plásticos, envases, mallas protectoras, metal, etc.) que son necesarios para estas explotaciones, cuyo objetivo es ser muy productivas en la menor superficie posible. Algunos ejemplos de estos cambios en la gestión agrícola son los cultivos bajo plástico en lo que fueron campos abandonados en Almería y donde se cultivan básicamente hortalizas. Pero también han visto una intensificación los nuevos cultivos de frutales en el interior de la provincia de Murcia y Alicante. En Valencia, la reactivación de esta agricultura es liderada por los cítricos, los cuales también son visibles en otras provincias de las costas mediterráneas desde Tarragona a Málaga, además de las grandes plantaciones desarrolladas en Huelva. En ocasiones conviven el abandono de oli- 
vares, almendros, frutales o viñedos con su transformación en invernaderos, nuevas plantaciones de cítricos, nuevos olivares en espaldera, o viñedos de nueva plantación. De estos últimos sabemos bien poco de su impacto sobre los procesos y tasas de erosión, y en caso de los cítricos la información aún es escasa (Cerdà y Giménez Morera, 2006; Cerdà et al., 2007, 2009a, 2009b; Giménez Morera y Cerdà, 2007; Giménez Morera et al., 2007).

En este artículo se presentan los resultados de la investigación llevada a cabo en las laderas de la vertiente norte de la Serra Grossa en la comarca de la Costera, provincia de Valencia. En esta zona se produjo el abandono de los campos de olivos, viñedos, almendros y frutales desde principios del siglo XX, pero sobretodo en los años 50 y los 60 . El viñedo fue la principal producción en los años 20 del siglo XX cuando la filoxera atacó los viñedos franceses, si bien siempre estuvo presente la diversificación de cultivos al ser necesaria para la agricultura de subsistencia y como estrategia comercial. En los años 60 fue el olivar, junto a los frutales y almendros, el cultivo más extendido. Esta zona muestra una evolución similar a la de los paisajes agrícolas de las zonas de media montaña mediterránea valenciana (Piqueras, 1999). Esos cultivos quedaron abandonados como consecuencia de los cambios económicos de finales de los años 50 y sobretodo los años 60 (Plan de Estabilización de 1959 y apertura del país al exterior) y la vegetación se recuperó, dando lugar a un denso matorral (Maquia), acompañada de la repoblación de pinos (Pinus halepensis). Eso favoreció que los incendios forestales fueran recurrentes, si bien la vegetación de matorral se recupera con éxito después de cada fuego, y determina que las pérdidas de suelo sean elevadas sólo en el año posterior a los incendios (Cerdà y Doerr, 2007). A partir de los años 90 se produce una colonización de estos campos por las nuevas plantaciones de cítricos, y con ello se alteran los procesos de erosión. La cuantificación de estos procesos erosivos a lo largo del tiempo no ha sido posible ya que hubiera supuesto mantener las mediciones durante más de medio siglo. Sin embargo, si es posible estimar como evolucionó la producción de sedimentos y aguas en campos de cultivo que aún conservan las características de los suelos durante el cultivo tradicional, los campos abandonados y los campos de cítricos actuales. Para ello se han seleccionado campos de cultivo que reproducen las condiciones de esas tres etapas de usos del suelo: cultivo tradicional de secano, abandono (0-5, 10-20 y > 30 años tras el abandono) y nuevas plantaciones de cítricos.

Los procesos erosivos están altamente condicionados por la intensidad de las precipitaciones. La exportación de sedimentos está determinada por las lluvias intensas, y no es extraño que más del $80 \%$ de la pérdida de suelo se produzca durante eventos extraordinarios (Romero Díaz et al., 1995; González-Hidalgo et al., 2009). Para poder cuantificar el impacto de estos eventos extraordinarios es necesario instalar y mantener parcelas, aforadores, piquetas de erosión u otros sistemas de medición continua (GarcíaRuiz y López-Bermúdez, 2009; Cerdà y Jordán, 2010) durante varias décadas. Para agilizar la investigación y como una primera aproximación se realizaron experimentos con lluvia simulada para conocer la respuesta de los suelos ante lluvias de intensidad elevada $\left(45.86 \mathrm{~mm} \mathrm{~h}^{-1}\right)$ que tienen en la zona de estudio una recurrencia de 5 años. Los experimentos se realizaron en parcelas de $12 \mathrm{~m}^{2}$ con el fin de cuantificar la erosión en zonas con la presencia de regueros ya que la erosión concentrada están presentes en las nuevas plantaciones de cítricos y en los campos recién abandonados. 
La lluvia simulada permite cuantificar la pérdida de suelo y agua, y para conseguir que estas sean representativas se requiere un estudio preliminar que debe mostrar las características de las superficies. Para ello, se realizaron transectos geomorfológicos que han permitido caracterizar las morfologías superficiales y estimar la actividad de los procesos erosivos.

El objetivo de este trabajo es mostrar el impacto que ha supuesto el uso agrícola tradicional, el abandono, y la posterior intensificación de la agricultura en los procesos erosivos en laderas del Este peninsular donde la llegada de estrategias de producción intensiva se viene produciendo desde los años 80 .

\section{Material y métodos}

\subsection{Zona de estudio}

Se seleccionó la vertiente norte de la Serra Grossa, la primera alineación montañosa del Sistema Bético, al sur de la provincia de Valencia, al existir en ella campos de secano tradicional en cultivo, campos abandonados y nuevas explotaciones intensivas de cítricos. Esto ha sido posible porque una parte de la población ha seguido manteniendo los campos agrícolas del secano tradicional por tradición y como complemento económico a su actividad económica principal en el sector secundario o terciario. El mantenimiento de estos campos ha sido posible también a la presencia de propietarios jubilados. Parte de los campos abandonados han sido adquiridos por empresas o particulares que han ejecutado transformaciones agresivas para la plantación de frutales, olivos en espaldera o viñedos, pero sobretodo de cítricos. La zona estudiada representa un espacio homogéneo del sur de los términos municipales de Montesa, Vallada, Moixent y Font de la Figuera, al pie de la Serra Grossa, sobre un substrato calcáreo de pendientes entre $2^{\circ}$ y $15^{\circ}$.

El clima de la zona es típico mediterráneo con una temperatura media anual de $16^{\circ} \mathrm{C}$ y veranos calurosos que han llegado a $47^{\circ} \mathrm{C}$ de temperatura máxima en tres ocasiones en los años 50, y $-10^{\circ} \mathrm{C}$ como mínima más baja en 2005 , en el cercano observatorio de Xàtiva. La precipitación media es de $500 \mathrm{~mm}$ anuales. La vegetación potencial es de encinares (Quercus ilex). El aprovechamiento histórico del encinar como fuente de carbón vegetal y leña lo hizo desaparecer. La vegetación predominante es la de matorral de Quercus coccifera, Pistacia lentiscus y Juniperus oxycedrus. En los años posteriores a los incendios son abundantes los jarales (Cistus albidus) pero también Rosmarinus officinalis, Thymus vulgaris, Ulex parviflorus y Fumana ericoides, además de Brachypodium retusum.

La explotación tradicional utilizó las zonas más agrestes como zonas de pastoreo, y las vaguadas y pies de ladera para el cultivo de secano. El regadío se localizaba junto a los manantiales o los pequeños azudes del Riu Canyoles, si bien la superficie ocupada fue ínfima respecto a la extensión de los cultivos de secano. Hoy, y fruto de la explotación del acuífero la agricultura de regadío se impone. 
A lo largo de la primera mitad del siglo XX se produjo la pérdida de población por migración, y en la segunda mitad el sector agrícola sigue perdiendo individuos por el traspaso de su población a otros sectores económicos. La pérdida de mano de obra supuso el abandono de los campos menos productivos, o los que pertenecían a familias que migraban, y con ello se iniciaba la regeneración vegetal, especialmente de matorral. A partir de 1990 se produce la recolonización de estas zonas abandonadas con nuevos cultivos intensivos de cítricos, los cuales aplican nuevas técnicas de manejo basadas en el uso de herbicidas.

\subsection{Métodos}

Los transectos geomorfológicos consisten en cuantificar que tipo de superficie se encuentra en contacto con la atmósfera. La metodología aplicada en este trabajo se ha basado en el trabajo pionero de Ruiz-Flaño et al. (1991), pero adaptados a las circunstancias y condiciones de la zona de estudio. En la práctica consiste en una cinta métrica tensada sobre el suelo, y sobre la que se cuantifica la superficie que tipo de cubierta o forma geomorfológica es predominante. Si bien los transectos geomorfológicos han sido abandonados por los estudios de erosión (García-Ruiz y López-Bermúdez, 2009), y no suelen aparecer en las revisiones sobre métodos en el estudio de la erosión del suelos o de la geomorfología (Cerdà y Jordán, 2010), es una herramienta de gran valor cuando se inicia el estudio de los procesos erosivos en una nueva zona de investigación. Así, la precisión es de $1 \mathrm{~cm}$, es decir, una vez extendida la cinta métrica se anota cada centímetro que tipo de superficie (la más representativa en ese centímetro) se encuentra en ese espacio. Se ha cuantificado la superficie que estaba afectada por costras, fragmentos de roca, vegetación, regueros (profundidad inferior a $10 \mathrm{~cm}$ ), cárcavas (profundidad superior a $10 \mathrm{~cm}$ ), y barrancos (profundidad superior a $100 \mathrm{~cm}$ ). Esta división se ha desarrollado a partir de las primeras visitas a las zonas de estudio y pruebas con el fin de mostrar todas las superficies existentes.

Los transectos geomorfológicos permiten cuantificar con sencillez los procesos erosivos, y además contribuyen a realizar un análisis espacial más exhaustivo que el estudio mediante parcelas de erosión o lluvia simulada, que representan una medición puntual de los procesos erosivos. La combinación de ambas métodos permite tener una visión amplia del problema (transectos) y una cuantificación rigurosa del proceso (lluvia simulada). Se seleccionaron 12 parcelas: 4 en campos de secano tradicional activos, 4 en campos abandonados menos de 5 años, 4 en campos abandonados entre 10 y 20 años, 4 parcelas en campos abandonados más de 30 años, y 4 en campos activos de nuevas plantaciones de cítricos. Cada parcela consistía en un campo de cultivo en el que se realizaban tres subtransectos de $100 \mathrm{~m}$ en la parte alta, $100 \mathrm{~m}$ la parte media y $100 \mathrm{~m}$ en la parte baja del campo. Los transectos se seleccionaban aleatoriamente pero siempre en sentido perpendicular a la pendiente, de manera que las formas de erosión lineales (regueros, cárcavas y barrancos) fuesen muestreados adecuadamente. La longitud de los sub-transectos era de 100 metros. Cuando en una parcela no se disponía de esa distancia en sentido perpendicular a la pendiente se continuaba el transecto en la parcelas colindante. Por lo tanto, se describió el tipo de morfología que se encontraba en la superficie y en cada una de las cinco 

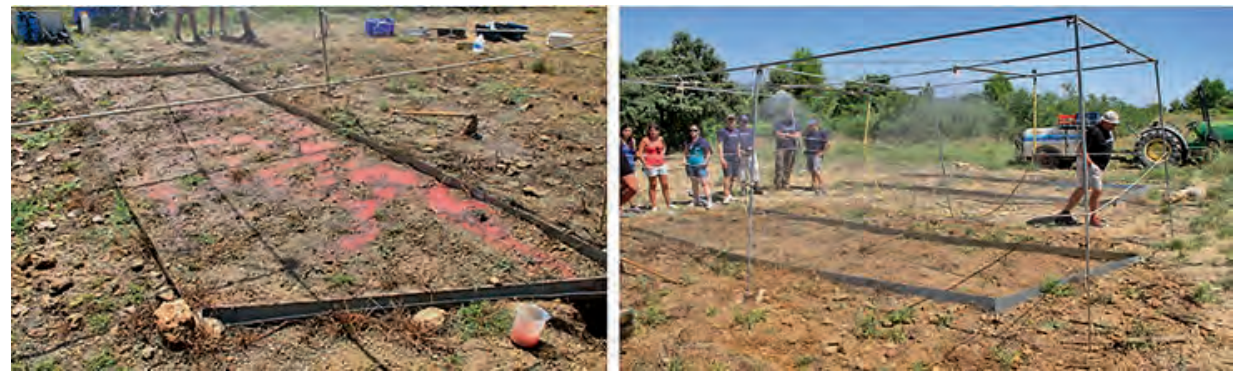

Figura 1. Vista de dos experimentos con la parcela de $12 \mathrm{~m}^{2}$ y el simulador de lluvia sin cubierta protectora del viento.

zonas seleccionadas se estudiaron 4 parcelas de $300 \mathrm{~m}$, lo que totaliza $6 \mathrm{Km}$ de mediciones (5 zonas x 4 parcelas x $300 \mathrm{~m}$ por parcela). Las mediciones se realizaron en agosto de 2008 con los suelos de cultivo tradicional de secano recién labrados y los suelos de explotaciones de cítricos tratados con herbicidas y con muy escasa cubierta vegetal. Tanto el laboreo en el olivar en secano, como herbicidas en el regadío de cítricos sirven para eliminar la competencia de las malas hierbas. En octubre de 2007 se produjo una precipitación intensa (180 $\left.\mathrm{mm} \mathrm{día}^{-1}\right)$ y que dejo en los campos de cítricos morfologías erosivas como regueros, cárcavas y barrancos. En aquellos que hubo laboreos los regueros, cárcavas y barrancos fueron eliminados por el paso del arado. En los campos de cítricos y en los campos abandonados menos de 5 años permanecieron hasta el momento del muestreo.

Los experimentos con lluvia simulada se realizaron sobre parcelas de $2 \mathrm{~m}$ de anchura y $6 \mathrm{~m}$ de longitud. Para conseguir una lluvia constante y homogénea se utilizaron dos boquillas lechler 461.008 siguiendo los protocolos presentados en Cerdà (1995). La calibración de laboratorio realizada en junio de 2008 mostró un coeficiente de Christiansen de $83.5 \%$ a una presión de 1.4 bares. La intensidad de las lluvias simulada fue de 45.86 $\mathrm{mm} \mathrm{h}^{-1}$ y se aplicó sobre una superficie de $35 \mathrm{~m}^{2}\left(12 \mathrm{~m}^{2}\right.$ de parcela y $23 \mathrm{~m}^{2}$ como zona de amortiguación). La lluvia se produjo a una altura de $2.3 \mathrm{~m}$, desde las dos boquillas instaladas sobre un soporte metálico (ver figura 1) y cubierto por un toldo protector. Las parcelas de $2 \mathrm{~m}$ x $6 \mathrm{~m}$ se delimitaron por medio de planchas de hierro galvanizado de 1 $\mathrm{mm}$ de grosor, $2000 \mathrm{~mm}$ longitud y $150 \mathrm{~mm}$ de altura (1 mm x $150 \mathrm{~mm}$ x $2000 \mathrm{~mm})$. El drenaje de la parcela se produjo a través de un canalón de $20 \mathrm{~cm}$ que comunicaba con tubo de $35 \mathrm{~mm}$ de diámetro, que vertía en un recipiente donde se colectaban las muestras de agua y escorrentía.

Las escorrentías se cuantificaban cada minuto, y cada cinco minutos se recogía una muestra para determinar la concentración de sedimentos. A partir del inicio de la lluvia se determinaba cuando se producía el primer encharcamiento y la escorrentía dentro de la parcela (ver Cerdà, 1996). La concentración de sedimentos se cuantificó tras la desecación de las muestras en laboratorio, y la humedad previa del suelo por gravimetría.

Para comparar las medias entre las diferentes tipos de parcelas muestreados se realizó un test ANOVA como análisis estadístico. Para conocer las diferencias entre las dife- 
rentes parcelas se utilizó el test de Tukey con un nivel de significancia de $\mathrm{P}<0.05$. El ajuste de los datos a una distribución Normal se llevó a cabo con el test de Kolmogorob-Smirnov. Los análisis estadísticos se realizaron con el SPSS 14 (ㄷ SPSS Inc, 1989).

\section{Resultados}

La zona de estudio presenta una evolución típica de las zonas de montaña media mediterránea en la que el abandono del campo fue constante a lo largo de los primeros 60 años del siglo XX, pero a diferencia de otros espacios más alejados de las zonas activas económicamente, la despoblación no fue total, sino que la cercanía a la costa (40-70 Km) y la ciudad de Valencia (70-100 Km) permitió el desarrollo de actividades industriales que mantuvieron la población en las zonas rurales. En la zona de estudio se encuentran dos municipios, Vallada y Moixent, en los que la población además se recuperó a partir de los años 60 fruto del asentamiento de una industria del mueble basada en el mimbre y el ratán, además de la explotación maderera centrada en Moixent, que permitió incluso aumentar la población. En Montesa, que siguió dependiendo de la actividad agrícola y de industrias localizadas fuera de su término municipal no se han encontrado cambios apreciables en el número de habitantes. En Font de la Figuera, el término municipal más alejado de la costa, la población se ha reducido claramente a lo largo del siglo XX (fig. 2).

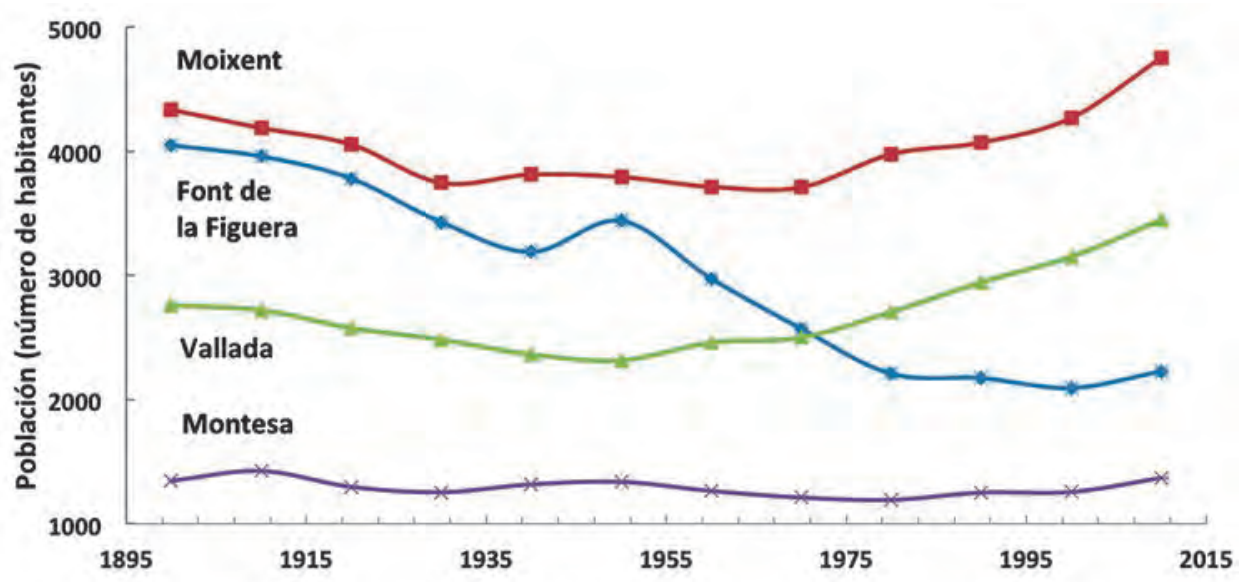

Figura 2. Evolución de la población en los municipios de la zonas de estudio desde 1990 a 2000.

Los transectos geomorfológicos muestran como se han modificado las formas superficiales y la cubierta vegetal con el abandono y con la recuperación e intensificación de la actividad agrícola (tabla 1). Durante el cultivo tradicional de secano las costras no estaban presentes a causa del laboreo, si bien tras las lluvias se extendían rápidamente como es visible en cualquier campo de cultivo de secano. No obstante, los agricultores labran el campo tan pronto como es posible para romper estas costras. 
Tabla 1. Transectos geomorfológicos en las cinco subzonas de estudio. Se han distinguido tres tipos de incisiones: regueros (profundidad inferior a $10 \mathrm{~cm}$ ), cárcavas (profundidad superior a $10 \mathrm{~cm}$ ), y barrancos (profundidad superior a $100 \mathrm{~cm}$ ).

\begin{tabular}{|c|c|c|c|c|c|c|c|}
\hline$(\%)$ & Costras & Piedras & Plantas & Regueros & Cárcavas & Barrancos & Labrado \\
\hline \multicolumn{8}{|c|}{ Cultivo secano } \\
\hline 1 & 0.00 & 6.85 & 0.35 & 0.00 & 0.00 & 0.00 & 92.80 \\
\hline 2 & 0.00 & 7.98 & 2.65 & 0.00 & 0.00 & 0.00 & 89.37 \\
\hline 3 & 0.00 & 10.25 & 4.25 & 0.00 & 0.00 & 0.00 & 85.50 \\
\hline 4 & 0.00 & 2.69 & 7.25 & 0.00 & 0.00 & 0.00 & 90.06 \\
\hline Media & 0.00 & 6.94 & 3.63 & 0.00 & 0.00 & 0.00 & 89.43 \\
\hline \multicolumn{8}{|c|}{ Abandono 0-5 } \\
\hline 1 & 66.68 & 15.65 & 15.32 & 2.35 & 0.00 & 0.00 & 0.00 \\
\hline 2 & 67.99 & 15.65 & 14.68 & 1.45 & 0.23 & 0.00 & 0.00 \\
\hline 3 & 62.41 & 15.65 & 19.25 & 2.69 & 0.00 & 0.00 & 0.00 \\
\hline 4 & 55.46 & 15.65 & 25.32 & 3.25 & 0.32 & 0.00 & 0.00 \\
\hline Media & 63.14 & 15.65 & 18.64 & 2.44 & 0.14 & 0.00 & 0.00 \\
\hline \multicolumn{8}{|c|}{ Abandono 10-20 } \\
\hline 1 & 10.25 & 10.25 & 78.95 & 0.20 & 0.35 & 0.00 & 0.00 \\
\hline 2 & 16.25 & 8.36 & 74.62 & 0.36 & 0.41 & 0.00 & 0.00 \\
\hline 3 & 14.02 & 4.58 & 80.74 & 0.15 & 0.26 & 0.25 & 0.00 \\
\hline 4 & 12.30 & 6.57 & 79.71 & 0.48 & 0.58 & 0.36 & 0.00 \\
\hline Media & 13.21 & 7.44 & 78.51 & 0.30 & 0.40 & 0.15 & 0.00 \\
\hline \multicolumn{8}{|c|}{ Abandono 30} \\
\hline 1 & 5.26 & 4.56 & 89.50 & 0.12 & 0.20 & 0.36 & 0.00 \\
\hline 2 & 4.23 & 5.68 & 89.12 & 0.15 & 0.30 & 0.52 & 0.00 \\
\hline 3 & 5.01 & 4.25 & 90.21 & 0.00 & 0.12 & 0.41 & 0.00 \\
\hline 4 & 2.35 & 3.25 & 93.73 & 0.23 & 0.09 & 0.35 & 0.00 \\
\hline Media & 4.21 & 4.44 & 90.64 & 0.13 & 0.18 & 0.41 & 0.00 \\
\hline \multicolumn{8}{|c|}{ Cítricos } \\
\hline 1 & 81.93 & 6.58 & 5.26 & 4.56 & 1.02 & 0.65 & 0.00 \\
\hline 2 & 83.79 & 4.98 & 4.25 & 5.32 & 1.06 & 0.60 & 0.00 \\
\hline 3 & 80.85 & 8.35 & 3.12 & 6.88 & 0.35 & 0.45 & 0.00 \\
\hline 4 & 81.5 & 9.24 & 4.20 & 4.20 & 0.32 & 0.54 & 0.00 \\
\hline Media & 82.02 & 7.29 & 4.21 & 5.24 & 0.69 & 0.56 & 0.00 \\
\hline
\end{tabular}

En los campos estudiados tras el laboreo se muestra como el suelo labrado cubre el $89.43 \%$ de la superficies, mientras que el resto corresponde a vegetación y hojarasca que queda en superficie $(3.63 \%)$ y fragmentos de roca $(6.92 \%)$. 
Cuando el campo se abandona, se desarrollan costras (63.14\%), aumentan las piedras en superficie (15.65\%), se forman regueros $(2.44 \%)$, y cárcavas $(0.14 \%)$, y también aparece una vegetación incipiente (18.64\%) de Brachypodium retusum, Fumana ericoides y Thymus vulgaris. Entre los 10 y 20 años de abandono los campos se cubren de plantas (78.51\%) con un extraordinario desarrollo de Rosmarinus officinalis, Cistus albidus, Ulex parviflorus y la generalización de la presencia de Brachypodium retusum, junto a las especies citadas anteriormente en la primera etapa de colonización. Las costras están presentes en las zonas entre matorrales (13.21\%) pero la cubierta de piedras decrece por la acumulación de hojarasca en superficie, si bien por debajo de esta se ha comprobado como el suelo se enriquece en fragmentos de rocas de forma que llegan a formar un enlosado. Los regueros se reducen $(0.30 \%$ de la superficie) pero aumentan las cárcavas $(0.40 \%)$ y aparecen algunos barrancos $(0.15 \%)$. Los campos abandonados más de 30 años presentan el $90.64 \%$ de su superficie cubierta de plantas u hojarasca, con sólo un $4.21 \%$ de costras, $4.44 \%$ de piedras, 0.13 de regueros, $0.18 \%$ de cárcavas y $0.41 \%$ de barrancos.

Las transformaciones agrícolas desarrolladas para ejecutar las nuevas plantaciones de cítricos destruyen las terrazas de cultivos antiguas e instalan el cultivo en pendiente. Tras el primer año de cultivo se produce el encostramiento del $82.02 \%$ de la superficie fruto del uso de herbicidas y la falta de vegetación que permiten el impacto de las gotas de lluvia. La vegetación sólo cubre el $4.21 \%$ del suelo, y se ha cuantificado un aumento de la pedregosidad superficial (7.29\%), la incipiente aparición de regueros $(5.24 \%)$, la formación de cárcavas $(0.69 \%)$ y la presencia de algunos barran$\cos (0.56 \%)$ que no han sido reparados por los agricultores desde las últimas lluvias de octubre de 2007, respecto a los campos antes de ser transformados en nuevas explotaciones.

Los experimentos con lluvia simulada han permitido determinar que en los suelos agrícolas del secano tradicional el encharcamiento y la escorrentía necesitan 617 y 1042 segundos respectivamente, y en término medio para las cuatro parcelas, de una lluvia de $45.86 \mathrm{~mm} \mathrm{~h}^{-1}$ para producirse (ver tabla 2). Estos experimentos se realizaron con suelos secos y labrados en verano con una humedad inferior al $5 \%$ entre 0 y $2 \mathrm{~cm}$ de profundidad. Los suelos abandonados recientemente (menos de 5 años) mostraban encharcamientos (258 s) y escorrentías (446 s) más rápidas. En las parcelas abandonadas 10-20 años y más de 30 años tanto el encharcamiento como la escorrentía se retrasaban hasta el punto de producirse tras 2766 y 3447 s respectivamente en las parcelas abandonadas más de 30 años. En los campos de cítricos, por el contrario, las escorrentías fueron muy rápidas (154 s) fruto de encharcamientos incipientes (105 s). La generación de escorrentía es muy rápida en los suelos cultivados con cítricos debido como lo demuestra el escaso tiempo transcurrido entre el encharcamiento y la escorrentía (49 s). En el caso de los suelos recién abandonados es de $188 \mathrm{~s}$, mientras que en los suelos abandonados más de 10 años o cultivados el retraso es mayor $(<400$ segundos) por la rugosidad producida por el laboreo o por la densa cubierta vegetal de los suelos abandonados. 
Tabla 2. Tiempo de encharcamiento (Tp), Tiempo de escorrentía (Tr) y diferencia entre el tiempo entre ambos. Tiempo en segundos a partir del inicio de la lluvia.

\begin{tabular}{|c|c|c|c|}
\hline Segundos & $T p$ & $\mathrm{Tr}$ & $T p-T r$ \\
\hline \multicolumn{4}{|c|}{ Cultivo secano } \\
\hline 1 & 569 & 998 & 429 \\
\hline 2 & 657 & 1068 & 411 \\
\hline 3 & 857 & 1206 & 349 \\
\hline 4 & 385 & 897 & 512 \\
\hline Media & 617 & 1042 & 425 \\
\hline \multicolumn{4}{|c|}{ Abandono 0-5 } \\
\hline 1 & 284 & 426 & 142 \\
\hline 2 & 269 & 468 & 199 \\
\hline 3 & 325 & 594 & 269 \\
\hline 4 & 153 & 296 & 143 \\
\hline Media & 258 & 446 & 188 \\
\hline \multicolumn{4}{|c|}{ Abandono 10-20 } \\
\hline 1 & 569 & 1039 & 470 \\
\hline 2 & 658 & 1110 & 452 \\
\hline 3 & 749 & 1460 & 711 \\
\hline 4 & 924 & 1520 & 596 \\
\hline Media & 725 & 1282 & 557 \\
\hline \multicolumn{4}{|c|}{ Abandono 30} \\
\hline 1 & 3020 & 3500 & 480 \\
\hline 2 & 2560 & 3068 & 508 \\
\hline 3 & 3300 & 3580 & 280 \\
\hline 4 & 2980 & 3450 & 470 \\
\hline Media & 2965 & 3400 & 435 \\
\hline \multicolumn{4}{|l|}{ Cítricos } \\
\hline 1 & 56 & 96 & 40 \\
\hline 2 & 98 & 114 & 16 \\
\hline 3 & 150 & 201 & 51 \\
\hline 4 & 116 & 204 & 88 \\
\hline Media & 105 & 154 & 49 \\
\hline
\end{tabular}

Después de 60 minutos de lluvia $(45.86 \mathrm{~mm})$ se registraron escorrentías del $7.06 \%$ de la lluvia en los campos de cultivo de secano, $16.49 \%$ en los recién abandonados, y de 1.77 y $0.4 \%$ en los abandonados entre 10 y 20 años y los que superaron los 30 años de aban- 
dono, respectivamente (tabla 3 y fig. 3a). Los suelos cultivados con cítricos alcanzaron un $50 \%$ de la lluvia transformada en caudal, donde se observan diferencias significativas. La concentración de sedimentos fue muy alta durante el cultivo de secano $\left(8.31 \mathrm{~g} \mathrm{l}^{-1}\right)$ y quedo en valores bajos (2.64 $\mathrm{g} \mathrm{l}^{-1}$ ) en los suelos abandonados (tabla 4). Los valores de la concentración de sedimentos alcanzan el valor mínimo en los suelos abandonados entre 10 y 20 años $\left(1.14 \mathrm{~g} \mathrm{l}^{-1}\right)$ y muy bajos en los abandonados más de 30 años $\left(0.23 \mathrm{~g} \mathrm{l}^{-1}\right)$ aunque no se observan diferencias estadísticamente significativas entre dichas parcelas (fig. 2). En el cultivo de cítricos la concentración de sedimentos es muy elevada: $20.46 \mathrm{~g} \mathrm{l}^{-1}$ de media, con una valor extremo medio en la parcela 3 con $30.25 \mathrm{~g} \mathrm{l}^{-1}$ (tabla $3 \mathrm{y}$ fig. $3 \mathrm{~b}$ ).

Tabla 3. Escorrentía, concentración de sedimentos y erosión en los 20 experimentos realizados en los 5 tipos de superficies en la vertiente norte de la Serra Grossa.

\begin{tabular}{|c|c|c|c|c|c|c|c|c|}
\hline & \multicolumn{3}{|c|}{ Escorrentía } & \multicolumn{3}{|c|}{ Sedimentos } & \multicolumn{2}{|c|}{ Erosión } \\
\hline & $\%$ & L & $\mathrm{mm}$ & $\mathrm{g} \mathrm{L}^{-1}$ & g & $\mathrm{g} \mathrm{m}^{2} \mathrm{~h}^{-1}$ & $\mathrm{Mg} \mathrm{ha}^{-1} \mathrm{~h}^{-1}$ & $\mathrm{Kg} \mathrm{ha}^{-1} h^{-1}$ \\
\hline \multicolumn{9}{|c|}{ Cultivo secano } \\
\hline 1 & 6.95 & 38.25 & 0.05 & 6.58 & 251.67 & 20.97 & 0.21 & 209.72 \\
\hline 2 & 7.86 & 43.26 & 0.06 & 10.58 & 457.64 & 38.14 & 0.38 & 381.37 \\
\hline 3 & 8.45 & 46.50 & 0.06 & 6.84 & 318.07 & 26.51 & 0.27 & 265.06 \\
\hline 4 & 4.96 & 27.30 & 0.04 & 9.25 & 252.49 & 21.04 & 0.21 & 210.41 \\
\hline Media & 7.06 & 38.83 & 0.05 & 8.31 & 319.97 & 26.66 & 0.27 & 266.64 \\
\hline \multicolumn{9}{|c|}{ Abandono 0-5 } \\
\hline 1 & 15.35 & 84.47 & 0.12 & 3.25 & 274.54 & 22.88 & 0.23 & 228.78 \\
\hline 2 & 16.48 & 90.69 & 0.13 & 2.48 & 224.92 & 18.74 & 0.19 & 187.43 \\
\hline 3 & 19.55 & 107.59 & 0.15 & 2.36 & 253.91 & 21.16 & 0.21 & 211.59 \\
\hline 4 & 14.56 & 80.13 & 0.11 & 2.47 & 197.91 & 16.49 & 0.16 & 164.93 \\
\hline Media & 16.49 & 90.72 & 0.13 & 2.64 & 237.82 & 19.82 & 0.20 & 198.18 \\
\hline \multicolumn{9}{|c|}{ Abandono 10-20 } \\
\hline 1 & 2.32 & 12.77 & 0.02 & 1.35 & 17.24 & 1.44 & 0.01 & 14.36 \\
\hline 2 & 2.51 & 13.81 & 0.02 & 0.98 & 13.54 & 1.13 & 0.01 & 11.28 \\
\hline 3 & 1.25 & 6.88 & 0.01 & 0.78 & 5.37 & 0.45 & 0.00 & 4.47 \\
\hline 4 & 0.99 & 5.45 & 0.01 & 1.45 & 7.90 & 0.66 & 0.01 & 6.58 \\
\hline Media & 1.77 & 9.73 & 0.01 & 1.14 & 11.01 & 0.92 & 0.01 & 9.17 \\
\hline \multicolumn{9}{|c|}{ Abandono 30} \\
\hline 1 & 0.00 & 0.00 & 0.00 & 0.00 & 0.00 & 0.00 & 0.00 & 0.00 \\
\hline 2 & 1.03 & 5.67 & 0.01 & 0.35 & 1.98 & 0.17 & 0.00 & 1.65 \\
\hline 3 & 0.00 & 0.00 & 0.00 & 0.00 & 0.00 & 0.00 & 0.00 & 0.00 \\
\hline 4 & 0.58 & 3.19 & 0.00 & 0.58 & 1.85 & 0.15 & 0.00 & 1.54 \\
\hline Media & 0.40 & 2.22 & 0.00 & 0.23 & 0.96 & 0.08 & 0.00 & 0.80 \\
\hline \multicolumn{9}{|c|}{ Cítricos } \\
\hline 1 & 60.25 & 331.57 & 0.46 & 15.32 & 5079.62 & 423.30 & 4.23 & 4233.02 \\
\hline 2 & 54.32 & 298.93 & 0.42 & 14.25 & 4259.81 & 354.98 & 3.55 & 3549.84 \\
\hline 3 & 39.58 & 217.82 & 0.30 & 30.25 & 6588.95 & 549.08 & 5.49 & 5490.79 \\
\hline 4 & 45.78 & 251.94 & 0.35 & 22.01 & 5545.12 & 462.09 & 4.62 & 4620.94 \\
\hline Media & 49.98 & 275.06 & 0.38 & 20.46 & 5368.38 & 447.36 & 4.47 & 4473.65 \\
\hline
\end{tabular}


Tabla 4. Correlaciones entre la escorrentía, la concentración de sedimentos y la pérdida de suelo. ** significativas $p<0.001$.

\begin{tabular}{|l|c|c|}
\hline & Sedimentos $\left(\mathrm{g} \mathrm{l}^{-\mathbf{1}}\right)$ & Erosión $_{\left(\mathbf{K g ~ h a}^{-\mathbf{1}} \mathbf{~ h}^{-\mathbf{1}}\right)}$ \\
\hline Escorrentía $(\%)$ & $0.77^{* *}$ & $0.90^{* *}$ \\
\hline Sedimentos $\left(\mathrm{g} \mathrm{l}^{-1}\right)$ & & $0.93^{* *}$ \\
\hline
\end{tabular}
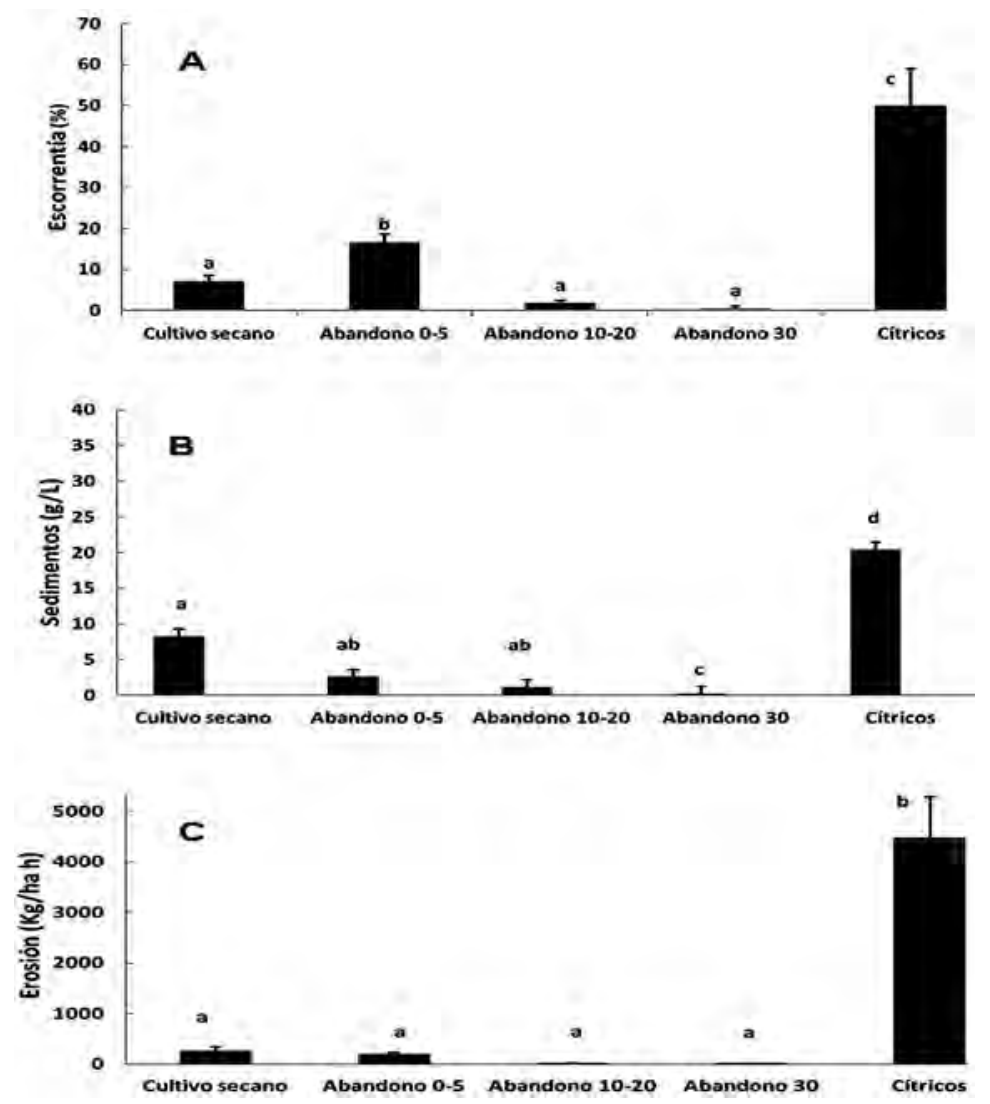

Figura 3. A.- Evolución de la escorrentía desde los cultivos de los años 50, al abandono y la intensificación de la agricultura a partir de los años 90 fruto de la implantación de cítricos. Letras distintas indican diferencias estadísticas significativas encontradas con el test de Tukey con un nivel de significancia de $P<0.05$. El ajuste de los datos a una distribución Normal se llevó a cabo con el test de Kolmogorob-Smirnov. B.- Evolución de la concentración de sedimentos desde los cultivos de los años 50, al abandono y la intensificación de la agricultura a partir de los años 90 fruto de la implantación de cítricos. Letras distintas indican diferencias estadísticas significativas encontradas con el test de Tukey con un nivel de significancia de P $<0.05$. El ajuste de los datos a una distribución

Normal se llevó a cabo con el test de Kolmogorob-Smirnov. C.- Evolución de la Erosion de suelo desde los cultivos de los años 50, al abandono y la intensificación de la agricultura a partir de los

años 90 fruto de la implantación de cítricos. Letras distintas indican diferencias estadísticas significativas encontradas con el test de Tukey con un nivel de significancia de $P<0.05$. El ajuste de

los datos a una distribución Normal se llevó a cabo con el test de Kolmogorob-Smirnov. 
La pérdida de suelo, fruto de los caudales y de la concentración de sedimentos en la escorrentías, muestra un patrón similar (tabla 4 y fig. 3c). Durante el cultivo hay una pérdida de 320 gramos de media para las parcelas de $12 \mathrm{~m}^{2}$, que se reducirán con el abandono: 238.11 y 1 gramo para los campos abandonados 0-5, 10-20 y más de 30 años. Esa reducción se verá alterada cuando se transforme el campo abandonado en un campo de cítricos, cuando se exportan 5368 gramos, siendo significativamente superior que en el resto de parcelas estudiadas. Las tasas de erosión fueron de $0.27 \mathrm{Mg} \mathrm{ha}^{-1} \mathrm{~h}^{-1}$ en el cultivo de secano, 0.20, 0.01 y $0 \mathrm{Mg} \mathrm{ha}^{-1} \mathrm{~h}^{-1}$ en los abandonados de 0-5, 10-20 y más de 30 años de abandono, respectivamente; mientras que en los campos de cítricos se produjo una tasa de erosión de $4.47 \mathrm{Mg} \mathrm{ha}^{-1} \mathrm{~h}^{-1}$.

\section{Discusión}

Los cambios en la actividad agrícola en España han hecho que algunas zonas sufrieran durante el siglo XX un intenso abandono de la agricultura tradicional (Arnáez et al., 2011), la cual se caracterizaba por un bajo nivel de insumos, en secano y con baja productividad (Naredo, 1996). Y que después se iniciara una recuperación de la actividad agrícola (1980-2010) muy localizada pero que ha permitido la llegada de una agricultura basada en los abonos químicos de síntesis, alta tecnificación, riego, pesticidas y herbicidas, y una visión industrial y comercial de la producción de alimentos. Estos cambios han supuesto una alteración del paisaje, y también de los procesos erosivos como se demuestra en este trabajo.

El cultivo tradicional fue, y debido fundamentalmente al continuo laboreo, muy esquilmante de suelo. Los trabajos realizados por el Instituto Pirenaico de Ecología han demostrado que ese impacto del cultivo se dejó sentir también a escala regional (GarcíaRuiz y Lasanta, 2004), pero sobre todo es evidente a escala de parcela (García-Ruiz et al., 1995; Lasanta et al., 2000; García-Ruiz, 2010), en la que otros grupos de investigación han aportado investigaciones de interés (Cerdà, 1997; Belmonte et al., 1999; Poyatos et al., 2003; Romero Díaz, 2003; Ruiz Sinoga, 2010). No obstante, esta tendencia a reducirse la pérdida de suelo puede no ser una norma general, ya que en zonas más áridas se ha comprobado una aumento de las tasas de erosión como muestran los regueros y las tubificaciones en campos de cultivos abandonados (Romero-Díaz et al., 2007).

En el sudeste de la Península Ibérica, por sus condiciones ambientales, esas altas tasas de erosión solo pudieron convivir con la producción agrícola gracias a la construcción de terrazas de cultivo que permitían retener y almacenar suelo. Estas terrazas de cultivo se han abandonado y sólo con la intensificación de la agricultura se han vuelto a su uso, si bien, habitualmente son destruidas para facilitar la mecanización de las parcelas. Ahora, y tras las transformaciones agrícolas las laderas presentan una pendiente continua y sin prácticas de conservación. Los trabajos de García-Orenes $(2009,2010)$ en la Estación Experimental de El Teularet-Sierra de Enguera, a $25 \mathrm{Km}$ de distancia de la zona de estudio, demostraron que los herbicidas dan lugar a una menor actividad microbiana, a agregados del suelo más inestables y con ello mayores tasas de erosión. 
El periodo crítico del abandono son los primeros años, y se ha demostrado aquí que es más productor de escorrentía que de sedimentos. Esto se debe a que los suelos se encostran y debido a la alta densidad de los suelos agrícolas y la formación de costras, favorece la formación de escorrentías abundantes. La formación de un enlosado de piedras como demostró Ruiz Flaño (1993) también reduce drásticamente la pérdida de suelos fruto de la reducción de la superficie expuesta a los procesos erosivos. Pero si los fragmentos de roca quedan apelmazados en el suelo, las escorrentías son muy abundantes (Cerdà, 2002). La pérdida de sedimentos se reduce al no existir sedimentos disponibles como si ocurría durante el cultivo, periodo en el que el continuo laboreo aumentaba la erodibilidad del suelo. El control que ejerce la concentración de sedimentos sobre las tasas de erosión lo podemos ver en la tabla 4, la cual es mayor al control que ejerce la producción de escorrentía.

A partir de los 10 años los suelos abandonados son muy estables y no aportan prácticamente sedimentos ni aguas a los cauces. En cambio, el flujo subsuperficial, el desarrollo de la hidrofobia (Bodí et al., 2012) y el flujo en macroporos son potenciados por una vegetación cada vez más densa y eficiente. Y muy probablemente en ese desarrollo de flujos preferentes a partir de la formación de superficies y zonas hidrofóbicas se deba a los incendios forestales (Arcenegui et al., 2010) que los favorecen (Granged, 2011).

El abandono del territorio supuso para las zonas de montaña la recuperación de la cubierta forestal, y con ello la estabilización de las pérdidas de agua y suelo. La investigación aquí presentada ha permitido comprobar cómo los primeros cinco años de abandono el suelo presenta elevadas pérdidas de agua al formase costras superficiales y ya no estar presente el agricultor para labrar y restaurar los campos cuando se formaban cárcavas y regueros. No obstante, en este caso, debido a la menor carga sedimentaria de las escorrentía generadas en el campo abandonado respecto al campo labrado, la pérdida de suelo se reduce en los primeros años de abandono. Y se reduce muy drásticamente tras 10-20 años, e incluso desaparece tras 30 años de abandono.

La intensificación de la agricultura con la citricultura ha supuesto la rotura de las terrazas de cultivo tradicional, la formación de grandes campos de cultivo sin sistema de drenaje y de control de la erosión, y el uso de herbicidas que han dado lugar a la pérdida de suelo por arroyadas superficiales.

La intensificación de la agricultura es una técnica propia de las zonas de regadío de zonas áridas y semiáridas. Un buen ejemplo de ellos son los cultivos en invernaderos en Almería que producen alimentos vegetales frescos para el norte de Europa. Pero también los cítricos, frutales, viñedos y olivares han aumentado su tecnificación y productividad debido al aumento de la fertilización, el riego y los productos fitosanitarios. El uso de herbicidas supone la reducción de la actividad microbiana, pérdida de cubierta vegetal y aumento de la pérdida de suelo (García-Orenes et al., 2009).

Este trabajo aporta información novedosa sobre el proceso de intensificación localizada de la agricultura industrializada. Los resultados demuestran que las tasas de erosión se disparan hasta tasas inusitadas debido a la eliminación de las terrazas de cultivo 
y a los manejos poco conservadores del suelo. Es por ello que será necesario desarrollar estrategias de control de la erosión y recuperación de los suelos que permitan desarrollar una agricultura sostenible. Algunos resultados muy esperanzadores se han encontrado con los acolchados de paja y el no laboreo con abonos verdes (García-Orenes et al., 2010) y con Geotextiles (Giménez Morera et al., 2010).

\section{Conclusiones}

Los resultados de la investigación aquí presentada demuestran que: (i) el cultivo tradicional generaba pérdidas de suelo y aguas elevadas; (ii) que las pérdidas de aguas se podían disparar durante los meses inmediatos al abandono por la formación de costras, escasa cubierta vegetal y la formación de una red de drenaje incipiente de regueros y algunas cárcava, pero que la pérdida de suelo se reducía por la menor concentración de sedimentos; (iii) que el abandono a partir de la primera década, y a pesar de los incendios, reduce la carga sedimentaria y las escorrentías hasta prácticamente hacer desaparecer la arroyada superficial; y que, (iv) el retorno de la actividad agrícola con técnicas intensivas supone el disparo de la pérdida de agua, y de suelo, hasta valores extraordinarios.

\section{Agradecimientos}

Los proyectos CGL2008-02879/BTE y LEDDRA 243857 financiaron esta investigación.

\section{Referencias bibliográficas}

Arcenegui, V., Mataix-Solera, J., Guerrero, C., Zornoza, R., Mayoral, A. M., MorALES, J. (2007). Factors controlling the water repellency induced by fire in calcareous Mediterranean forest soils. European Journal of Soil Science, 58: 1254-1259.

Arnáez, J., Lasanta, T., Errea, M. P., Ortigosa, L. (2011). Land abandonment, landscape evolution and soil erosion in a Spanish Mediterranean mountain region: the case of Camero Viejo. Land Degradation and Development, 22: 537-550.

Belmonte Serrato, F., Romero Díaz, A., López Bermúdez, F. (1999). Efectos sobre la cubierta vegetal, la escorrentía y la erosión del suelo, de la alternancia cultivoabandono en parcelas experimentales. Investigaciones Geográficas, 22: 95-107.

Bodí, M. B., Cerdà, A., MataiX-Solera, J., Doerr, S. H. (2012). Repelencia al agua en suelos forestales afectados por incendios y en suelo agrícolas bajo distintos manejos y abandono. Cuadernos de Investigación Geográfica, 38 (2): 53-74.

Bonet, A. (2004). Secondary succession of semi-arid Mediterranean old fields in southeastern Spain: insights for conservation and restoration of degraded lands. Journal of Arid Environments, 56: 203-233. 
Cammeraat, L. H., Imeson, A. C. (1999). The evolution and significance of soil-vegetation patterns following land abandonment and fire in Spain. Catena, 37: 107-127

Cerdè, A. (1995). Soil moisture regime under simulated rainfall in a three years abandoned field in Southeast Spain. Physics and Chemistry of the Earth, 20: 271-279.

CERDÀ, A. (1997). Soil erosion after land abandonment in a semi-arid environment of South eastern Spain. Arid Soil Research and Rehabilitation, 11: 163-176.

CERDÀ, A. (2001). Effects of rock fragment on soil infiltration, inter-rill runoff and erosion. European Journal of Soil Science, 52: 1-10.

Cerdì, A., Lasanta, A. (2005). Long-term erosional responses after fire in the Central Spanish Pyrenees: 1. Water and sediment yield. Catena, 60: 59-80.

Cerdà, A., Giménez Morera, A. (2006). Sostenibilidad y Medio Ambiente. En: La investigación ante la sociedad del conocimiento (Amat, M., Ed.), pp. 241-245.

Cerdè, A., Doerr, S. H. (2007). Soil wettability, runoff and erodibility of major dry Mediterranean land use types on calcareous soils. Hydrological Processes, 21: 2325-2336.

Cerdà, A., Jordán, A. (2010). Actualización en métodos y técnicas para el estudio de los suelos afectados por incendios forestales. Cátedra de Divulgació de la Ciència, Universitat de València, 589 pp., Valencia.

Cerdà, A., Bodí, M. B., Hevilla-Cucarella, E. B. (2007). Erosión del suelo en plantaciones de cítricos en laderas. Valle del riu Canyoles, Valencia. Agroecología, 2: $12-33$

Cerdà, A., Bodí, M. B., Segura, M., Giménez-Morera, A., Domínguez, A. (2009). Erosión del suelo en los "nuevos" naranjales valencianos ante lluvias torrenciales. Cuadernos de la Investigación en la Ingeniería, 73-86.

Collantes, F. (2001a). El declive demográfico de la montaña española, 1860-1991: revisión crítica de propuestas teóricas. Historia Agraria, 24: 203-225.

Collantes, F. (2004). La evolución de la actividad agrícola en las áreas de montaña españolas (1860-2000). Estudios Agrosociales y Pesqueros, 201: 79-104.

CRuZ, J. (1990). Les comarques de muntanya. Alfons el Magnànim, Valencia.

Debussche, M., LePART, J. (1992). Establishment of woody plants in Mediterranean old fields: opportunity in space and time. Landscape Ecology, 6: 133-145.

Debussche, M., Lepart, J., Dervieux, A. (1999). Mediterranean landscape changes: evidence from old postcards. Global Ecology and Biogeography, 8: 3-15.

Douglas, T. D., Kirkby, S. J., CRitchley, R. W., PARK, G. J. (1994). Agricultural terrace abandonement in the Alpujarra, Andalucia, Spain. Land Degradation \& Rehabilitation, 5: 281-291. 
García-Fayos, P., Verdú, M. (1998). Soil seed bank, factor controlling germination and establishment of a Mediterranean shrub- Pistacia lentiscus L. Acta OecologicaInternational Journal of Ecology, 19: 357-366.

García-Ruiz, J. M. (2010). The effects of land uses on soil erosion in Spain: A review. Catena, 81: 1-11.

García-Ruiz, J. M., Lasanta, T., eds. (1994). Efectos Geomorfológicos del Abandono de tierras. Sociedad Española de Geomorfología, 171 pp., Zaragoza.

García-Ruiz, J. M., Valero, B. (1998). Historical geomorphic processes and human activities in the Central Spanish Pyrenees. Mountain Research and Development, 18: 309-320.

García-Ruiz, J. M., LóPez-Bermúdez, F. (2009). La erosión del suelo en España. Sociedad Española de Geomorfología, 471 pp., Zaragoza.

García-Ruiz, J. M., Lasanta, T., Ortigosa, L., Ruiz-Flaño, P., Martí, C., González, C. (1995). Sediment yield under different land uses in the Spanish Pyrenees. Mountain Research and Development, 15: 229-240.

García-Ruiz, J. M., Arnáez, J., Beguería, S., Seeger, M., Martí-Bono, C., Regüés, D., Lana-Renault, N., White, S. (2005). Runoff generation in an intensively disturbed, abandoned farmland catchment, Central Spanish Pyrenees. Catena, 59: 79-92.

García-Orenes, F., Cerdà, A., Mataix-Solera, J., Guerrero, C., Bodí, M. B., ArceNEGUi, V., ZornOZA, R., SEMPERE, J. G. (2009). Effects of agricultural management on surface soil properties and soil-water losses in eastern Spain. Soil \& Tillage Research, 106: 117-123.

García-Orenes, F., Guerrero, C., Roldán, A., MataiX-Solera, J., Cerdà, A., Campoy, M., Zornoza, R., Bárcenas, G., Caravaca, F. (2010). Soil microbial biomass and activity under different agricultural management systems in a semiarid Mediterranean agroecosystem. Soil \& Tillage Research, 109: 110-115.

Garrabou, R., Barciela, C., Jiménez Blanco, J. I. (1986). Historia agraria de la España contemporánea. 3. El fin de la agricultura tradicional (1900-1960). Crítica, Barcelona.

Giménez Morera, A., Cerdà, A. (2007): Cambios ambientales y económicos en la citricultura. El ejemplo del regadío tradicional del Riu dels Sants en la huerta de Xàtiva. Cuadernos de Investigación de la Ingeniería, 307- 320.

Giménez Morera, A., Bodí, M. B., Cerdì. A. (2007). Agrotextiles en citricultura. Viabilidad económica, ambiental y agronómica en agricultura ecológica. En: La investigación ante la sociedad del conocimiento. Sostenibilidad y Medio Ambiente (Amat, M., Ed.), 111-116.

González-Hidalgo, J. C., Batalla, R. J., Cerdà, A., De Luis, M. (2009). Contribution of the largest events to suspended sediment transport across the USA. Land Degradation and Development, 21: 83-91. 
Granged. A. P., Jordán, A., Zavala, L. M., Bárcenas, G. (2011). Induced changes in soil water repellency increased fingered flow and runoff rates following the 2004 Huelva wildfire. Hydrological Processes, 25: 1614-1629.

Koulouri, M., Giourga, C. H. R. (2007). Land abandonment and slope gradient as key factor of soil erosion in Mediterranean terraced lands. Catena, 69: 274-281.

LASANTA, T. (1989). La evolución reciente de la agricultura de montaña: el Pirineo aragonés. Geoforma Ediciones, 220 pp., Logroño.

Lasanta, A., Cerdà, A. (2005). Long-term erosional responses after fire in the Central Spanish Pyrenees: 2. Solute release. Catena, 60: 80-101.

Lasanta, T., García-Ruiz, J. M., Pérez Rontomé, C., Sancho, C. (2000). Runoff and sediment yield in a semi-arid environment: The effect of land management after farmland abandonment. Catena, 38: 265-278.

Lasanta, T., Beguería, S., García Ruiz, J. M. (2006). Geomorphic and hydrological effects of traditional shifting agriculture in a Mediterranean mountain, Central Spanish Pyrenees. Mountain Research and Development, 26: 146-152.

Lasanta, T., Vicente-Serrano, S. M. (2007). Cambios en la cubierta vegetal en el Pirineo Aragonés en los últimos 50 años. Pirineos, 162: 125-1254.

MCNeILl, J. R. (1992). The Mountains of the Mediterranean World: a environmental history. Cambridge University Press, Cambridge, 456 pp.

NAREdo, J. M. (1996). La evolución de la agricultura en España (1940-1990). Universidad de Granada, 324 pp., Granada.

NE'EMAN, G., IzHAKI, I. (1996). Colonization in an abandoned East-Mediterranean vineyard. Journal of Vegetation Science, 7: 465-472.

Piqueras, J. (1999). El espacio Geográfico valenciano. Una síntesis geográfica, 356 pp., Valencia.

Poyatos, R., LAtron, J., Llorens, P. (2003). Land use and land cover change after agricultural abandonment. Mountain Research and Development, 23: 362-368

Romero-Díaz, A. (2003). Influencia de la litología en las consecuencias del abandono de tierras de cultivo en medios mediterráneos semiáridos. Papeles de Geografía, 38: $151-165$.

Romero-Diaz, A., BarberÁ, G. G., López Bermúdez, F. (1995). Relaciones entre erosión del suelo, precipitación y cubierta vegetal en un medio semiárido del sureste de la península ibérica. Lurralde, 18: 229-243.

Romero-Díaz, A., Marín-Sanleandro, P., SÁnchez-Soriano, A., Belmonte-SerraTO, F., FAULKNER, H. (2007). The causes of piping in a set of abandoned agricultural terraces in Southeast Spain. Catena, 69: 282-293.

Ruiz Flaño, P. (1993). Procesos de erosión en campos abandonados del Pirineo. El ejemplo del valle de Aísa. Geoforma Ediciones, 191 pp., Logroño. 
Ruiz-Flaño, P., Lasanta, T., García-Ruiz, J. M., Ortigosa, L. (1991). The diversity of sediment yield from abandoned fields of the Central Spanish Pyrenees. IAHS Publication, 203: 103-110.

Ruiz-Sinoga, J. D., Martínez-Murillo, J. F., Gabarrón-Galeote, M. A., GarcíaMARÍn, F. (2010). The effects of soil moisture variability on the vegetation pattern in Mediterranean abandoned fields (Southern Spain). Catena, 85: 1-11.

Vicente-Serrano, S. M., Lasanta, T., Cuadrat, J. M. (2000). Transformaciones en el paisaje del Pirineo como consecuencia del abandono de las actividades económicas tradicionales. Pirineos, 155: 111-113.

Zavala, L. M., Jordán, A., Bellinfante, N., Gil, J. (2010). Relationships between rock fragment cover and soil hydrological response in a Mediterranean environment. Soil Science and Plant Nutrition, 56: 95-104. 